\title{
Anatomical Evaluation of Lumbar Arteries for Lateral Lumbar Interbody Fusion with Magnetic Resonance Imaging
}

\author{
Masato Kiyohara, Takeshi Arizono, Akihiko Inokuchi, Takahiro Hamada, Kenjiro Nishida and Ryuta Imamura \\ Department of Orthopaedic Surgery, Kyushu Central Hospital of the Mutual Aid Association of Public School Teachers, \\ Fukuoka, Japan
}

\begin{abstract}
:
Introduction: Lateral lumbar interbody fusion (LLIF) is becoming a more common surgical treatment option for adult degenerative lumbar conditions. LLIF is a mini-open access technique with wound retractors, and postoperative hematoma due to segmental vessels injury is reported. Thus, it is considered that there is a need to conduct detailed preoperative examinations to identify where the lumbar vessels are. As far as we know, there are only a few studies investigating the location of the lumbar arteries. This study evaluates the anatomical position of lumbar arteries using magnetic resonance imaging (MRI).
\end{abstract}

Methods: We studied 101 MRIs of patients with lumbar disease. The length from the upper and lower end plates of the vertebra to the lumbar arteries was measured. The measurement was conducted with coronal MRI images of every quarter slice of L1 to L4 vertebrae. We also investigated sagittal MRI images to determine whether the lumbar vessels are located on intervertebral disc in each level from L1/2 to L5/S1.

Results: The lumbar vessels are not always located at the center of the vertebrae. Some lumbar vessels are located within $8 \mathrm{~mm}$ from the end plates. Especially in L4, the lumbar vessels tended to go down from the anterior cranial side to the posterior caudal side $(\mathrm{P}<0.01) .8,24$, and 54 lumbar vessels are located at the anterior quarter, the center, and the posterior quarter slice of the vertebrae, respectively, in L4. There were seven lumbar vessels in total located on the vertebral disc level.

Conclusions: It is necessary to investigate where the lumbar arteries are located to prevent its injury in LLIF, because the lumbar artery is not always located at the center of a vertebra. MRIs may provide a valuable information to avoid vascular injury during LLIF.

\section{Keywords:}

Lumbar spine, Degenerative lumbar disease, Lateral lumbar interbody fusion, Lumbar segmental vessels

Spine Surg Relat Res 2020; 4(1): 69-73 dx.doi.org/10.22603/ssrr.2019-0013

\section{Introduction}

Lateral lumbar interbody fusion (LLIF) is becoming a more common surgical treatment option for degenerative lumbar diseases. LLIF is a less invasive mini-open access technique with wound retractors. However, it is very important to understand the visceral anatomy including vessels in detail because of having a small portal.

Although segmental vessels are not usually identified during LLIF, there are some reports of segmental vessel lacerations ${ }^{1)}$. It is also reported that some major vascular complications such as abdominal aorta lacerations and common iliac vein lacerations are present ${ }^{1-3)}$. Rodgers et al. prospectively investigated 600 patients who had extreme lateral interbody fusion and reported that there were zero complications due to perioperative vascular injury ${ }^{4)}$. On the other hand, Mehren and colleagues reported vascular injury in $0.37 \%$ (3 out of 812 patients) in oblique lateral interbody fusion (OLIF) surgeries, 2 in the left common iliac vein and 1 aortic injury, at the L5 level ${ }^{2}$. Clément Silvestre et al. reported that 2 cases of iliac vein injury and 1 case of aortic injury were observed in 179 patients who underwent $\mathrm{OLIF}^{33}$.

These are markedly reduced risks of vascular injury as compared to those with conventional anterior approaches

Corresponding author: Masato Kiyohara, m-kiyo@ortho.med.kyushu-u.ac.jp

Received: March 1, 2019, Accepted: July 24, 2019, Advance Publication: September 20, 2019

Copyright (C) 2020 The Japanese Society for Spine Surgery and Related Research 
Table 1. Demographic Data for the Subjects.

\begin{tabular}{lc}
\hline Total subjects (n) & 101 \\
Sex (male/female) & $66 / 35$ \\
Age at time of operation (years) & Male, 57.9 (range, 27-84) \\
& Female, 63.2 (range, 17-88) \\
Group (n) & LCS, 56; LDH, 42; LS, 3 \\
\hline
\end{tabular}

n, number; LCS, lumbar canal stenosis; LDH, lumbar disc herniation; LS, lumbar spondylolisthesis

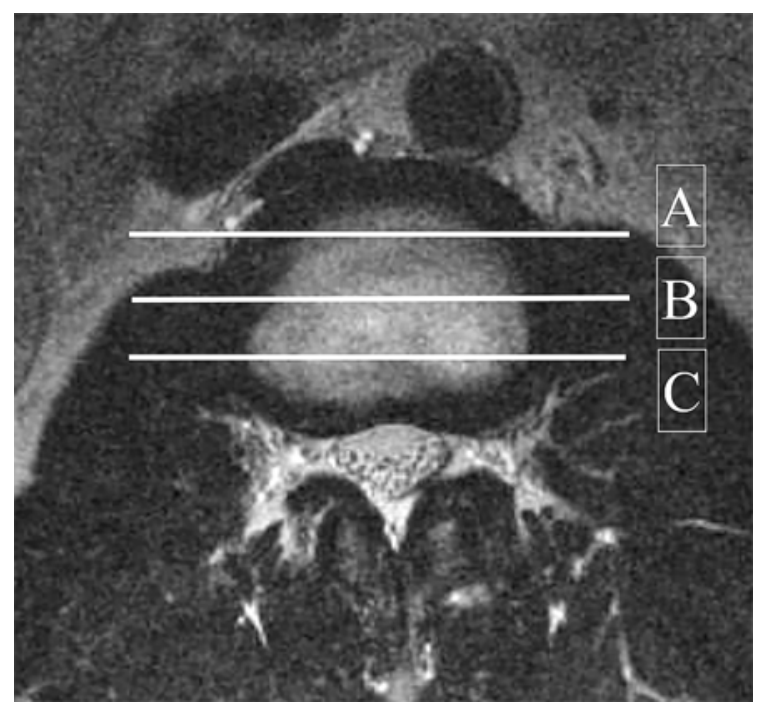

Figure 1. This figure shows the axial T2 MRI image. (A) Anterior quarter slice, (B) center slice, (C) posterior quarter slice

with $1.4 \%$ to $15.6 \%$ reports of a number of vascular injuries $^{5-10)}$. Although there is a greatly reduced risk of injury, it is considered that there is a need to conduct detailed preoperative examinations because vascular injury can be fatal. As far as we know, there are few studies investigating the location of the lumbar arteries.

We therefore considered that it is important to know the exact location of the lumbar arteries before the operation. We also investigated the position of the lumbar arteries with routinely obtained magnetic resonance imaging (MRI) before surgery.

\section{Materials and Methods}

We retrospectively analyzed data obtained from patients with lumbar spine disease who underwent preoperative MRI at our hospital between October 2014 and June 2015. Initially, 104 patients were included; however, 3 patients who had severe vertebral collapse or severe deformities (local scoliosis with more than a Cobb's angle of 10 degrees) were excluded. Finally, a total of 101 patients (66 males, 35 females) were included. The mean age of males was 57.9 years (range, 27-84); for females, it was 63.2 years of age (range, 17-88). 56 patients had lumbar canal stenosis, 42 patients had lumbar disc herniation, and 3 patients had lumbar

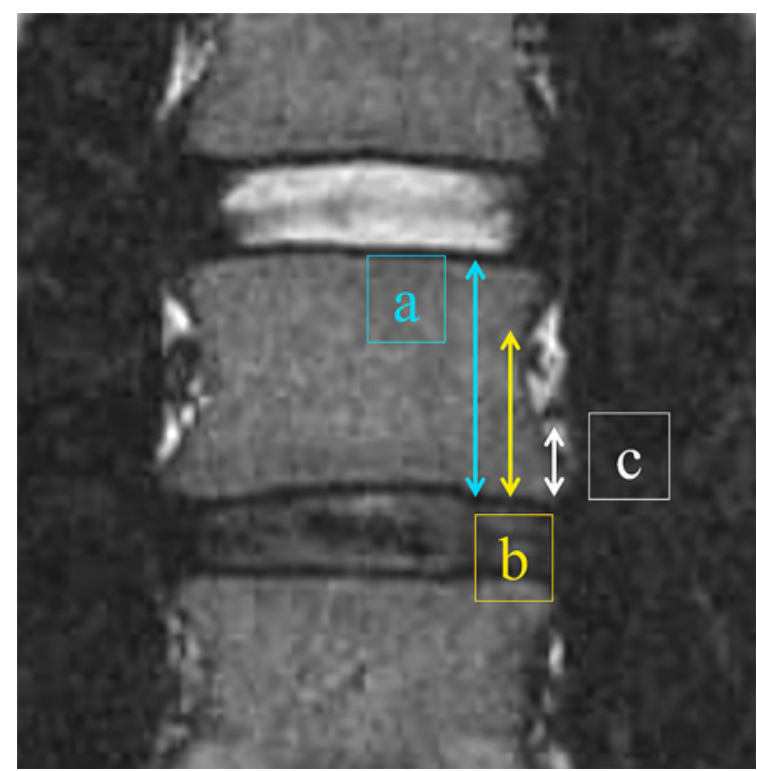

Figure 2. This figure shows the coronal T2 MRI image.

(a) Total length of the vertebra, (b) length from the lower end plate to the upper rim of lumbar vessels, (c) length from the lower end plate to the lower rim of lumbar vessels

spondylolisthesis of L5 (Table 1).

We divided a vertebral body into quarter slices in MRI axial images from L1 to L5 (Fig. 1). In each slice of the coronal image from the MRI, the length of the vertebra and the length from the lower end plates of the vertebra to the upper and lower rims of the lumbar vessels were measured (Fig. 2). The coronal slices were set at the perpendicular plane to the tangential line of the end plate in each vertebra. The measurement was conducted on MRI coronal images of every quarter slice of the L1 to L4 vertebrae of the lengths of the left side of the vertebrae. Calculation was done to evaluate the location of vessels with the value obtained by dividing "the length from the lower end plate of the vertebra to the upper rims of the lumbar vessels" by "the length of the vertebra" and dividing "the length from the lower end plates of the vertebra to the lower rims of the lumbar vessels" by "the length of the vertebra." We also investigated sagittal MRI images to determine whether the lumbar vessels are located on the intervertebral disc in each level from L1/2 to L5/S1 (Fig. 3). Then we recorded their axial position if they are found to be located on the intervertebral disc.

\section{Sources of equipment and statistical analysis}

All MRI studies were performed on a 1.5-Tesla unit (Magnetom Avanto, Siemens, Germany). Imaging protocols were T2-weighted fast spin-echo images (TR range/TE range, 4,000/94; slice thickness, $3 \mathrm{~mm}$ ) in the coronal or axial plane and STIR images (TR/TE, 5,800/90; inversion time, $160 \mathrm{msec}$; slice thickness, $3 \mathrm{~mm}$ ) in the sagittal plane. We used statistical software to perform the statistical analy- 
sis (R3.0.3; R Foundation for Statistical Computing, Austria $)^{11}$. The comparison among the lumbar vessel positions was made using the ANOVA test and Bonferroni test.

\section{Results}

In this present study, the lumbar vessels presented between $14 \%$ and $82 \%$ from the lower end plates on the lateral aspects of the vertebral body (Table 2). The standard deviations of the lumbar vessels' edges ranged from $7 \%$ to $14 \%$; the lumbar vessels are not always located at the center of the vertebrae. Especially at L4, the upper rims of the lumbar vessels were $74 \pm 8 \%$, and the lower rims of the lumbar vessels were $40 \pm 9 \%$ at the anterior quarter slice of the vertebrae, and at the center slice of the vertebral body, the upper rims of the lumbar vessels were $69 \pm 8 \%$, and the lower rims of the lumbar vessels were $34 \pm 9 \%$. At the posterior quarter slice of the vertebrae, the upper rims of the lumbar vessels were $66 \pm 14 \%$, and the lower rims of the lumbar vessels were $25 \pm 11 \%$. Compared with other vertebrae, in L4, the lumbar vessels tended to go down from the anterior cranial side to the posterior caudal side $(\mathrm{P}<0.01)$ (Fig. 4).

Some lumbar vessels are located within $8 \mathrm{~mm}$ from the
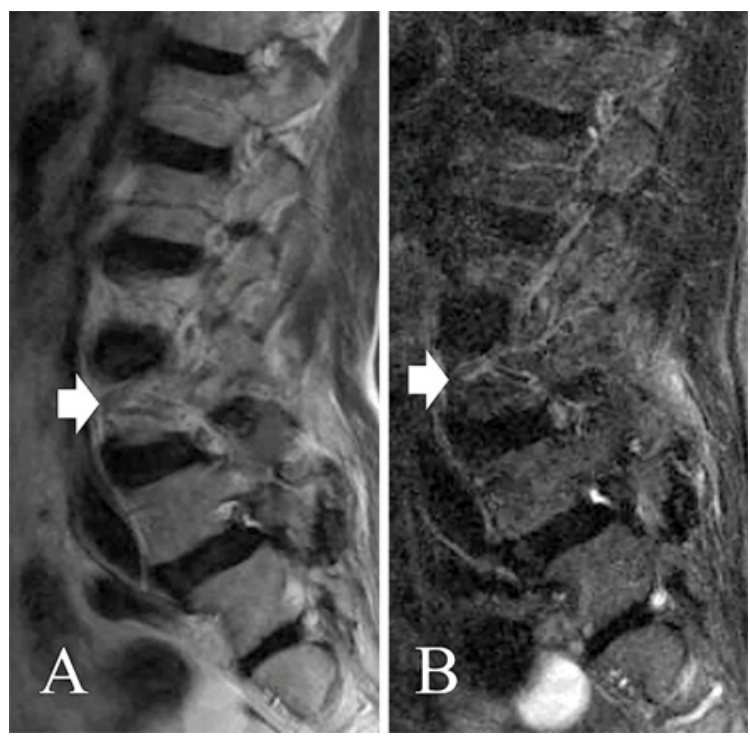

Figure 3. These figures show MRI images of one of the subjects, an 83-year-old male with lumbar stenosis. (A) Sagittal T2 image, (B) sagittal STIR image. The arrows indicate the right lumbar vessels located on L3/4. end plates. Thirty-one lumbar vessels are located at the anterior quarter slice of the vertebrae in L1; 38, 18, and 27 lumbar vessels are located at the anterior quarter slice, the center slice, and the posterior quarter slice of the vertebrae, respectively, in L2; 6, 11, and 36 vessels are located, respectively, in L3; and 8, 24, and 54 lumbar vessels are located, respectively, in $\mathrm{L} 4$.

There were also seven lumbar vessels located on the vertebral disc level using sagittal MRI images. The number of occurrences was 1, 2, 3, and 1 for L1/2, L2/3, L3/4, and L5/ S1 level, respectively. Only one lumbar vessel for the L3/4 level is located at the anterior quarter slice of the vertebra; all of the others are located at the posterior quarter slice of the vertebrae.

\section{Discussion}

The most remarkable finding of the current study was that the segmental arteriovenous vein did not necessarily run in the center of the vertebral body, and individual differences were observed. Especially at L4, it exists on the proximal side in the anterior quarter of the vertebral body and tended to exist on the distal side in the posterior quarter of the vertebral body. A number of lumbar vessels are located on the vertebral disc level, and quite a few lumbar vessels are also located within $8 \mathrm{~mm}$ from the end plates of the vertebrae.

Anatomically, the lumbar vessels branch off from the aorta, and it is generally understood that they travel down the side of the vertebral body, pass under the transverse process, and enter the intervertebral foramen ${ }^{12,13)}$. Orita et al. retrospectively evaluated the anatomical features of lumbar segmental arteries by using MRI, and they found that segmental arteries could be involved in the surgical field of OLIF especially in L4 and L5 levels ${ }^{14}$. In contrast with the former study, this study finds that several lumbar vessels are located on the vertebral disc level, and some lumbar vessels are also located within $8 \mathrm{~mm}$ from the end plates of the vertebrae. Especially at L4, more than $50 \%$ of the lumbar vessels $(54 / 101)$ exist within $8 \mathrm{~mm}$ from the end plate in the posterior quarter of the vertebral body.

The discrepancy between the former study and this present study might be due to the differences of patient characteristics and the way of measurement of the lumbar vessels. Further studies are needed to reveal why the difference occurred.

In OLIF, a screw to fix a retractor is designed located

Table 2.

\begin{tabular}{ccccccccccccc}
\hline & \multicolumn{3}{c}{ L1 } & \multicolumn{3}{c}{ L2 } & \multicolumn{1}{c}{ L3 } & \multicolumn{3}{c}{ L4 } \\
\cline { 2 - 13 } & A & C & P & A & C & P & A & C & P & A & C & P \\
\hline Upper (\%) & $63 \pm 11$ & $71 \pm 9$ & $71 \pm 7$ & $65 \pm 9$ & $71 \pm 9$ & $70 \pm 7$ & $73 \pm 7$ & $72 \pm 8$ & $69 \pm 8$ & $74 \pm 8$ & $69 \pm 8$ & $66 \pm 14$ \\
Lower (\%) & $32 \pm 10$ & $36 \pm 11$ & $37 \pm 9$ & $31 \pm 10$ & $35 \pm 9$ & $32 \pm 10$ & $40 \pm 7$ & $37 \pm 9$ & $30 \pm 10$ & $40 \pm 9$ & $34 \pm 9$ & $25 \pm 11$ \\
\hline
\end{tabular}

The means and standard deviations

$\mathrm{A}$, anterior quarter slice; $\mathrm{C}$, center slice; $\mathrm{P}$, posterior quarter slice 


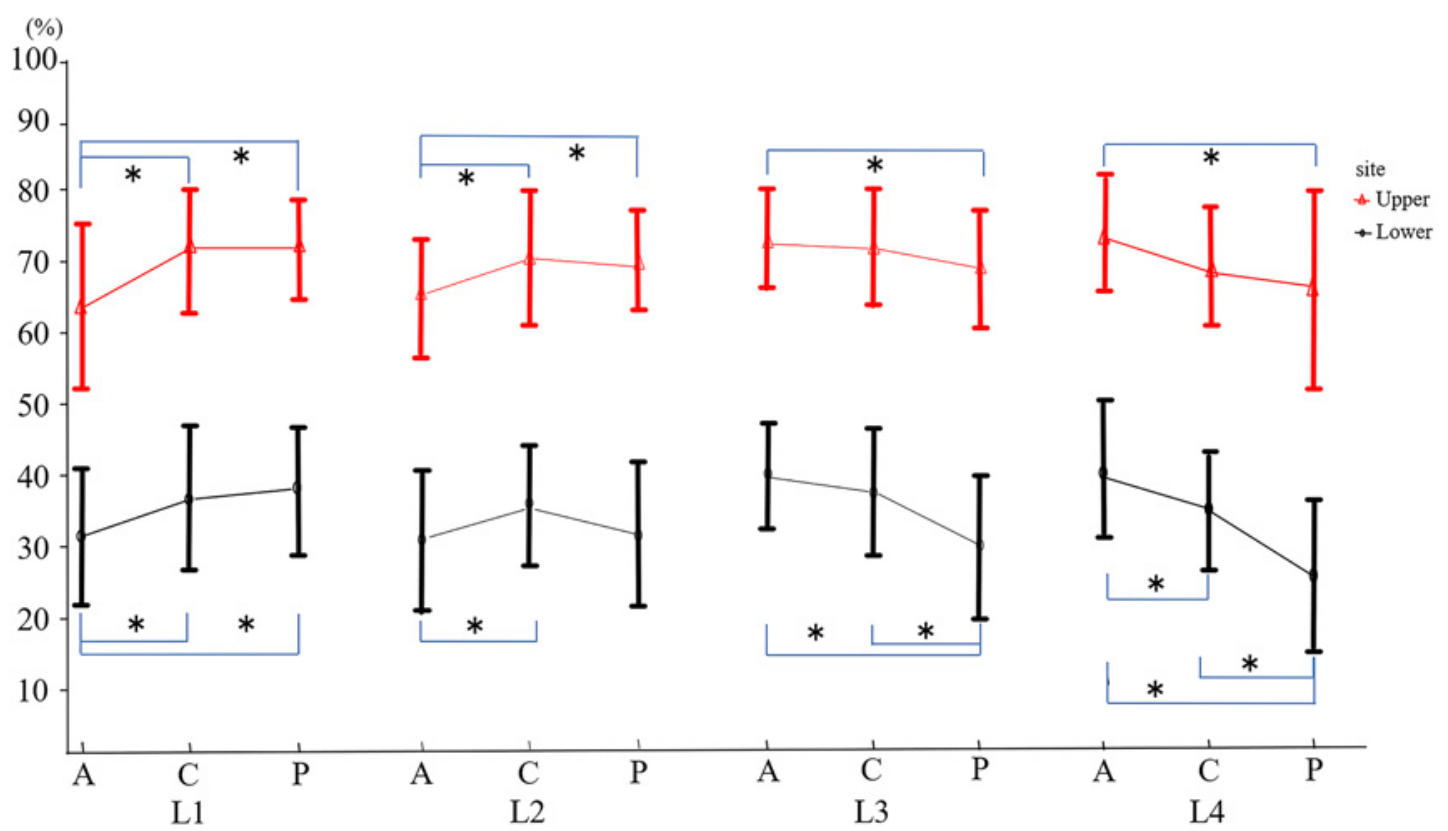

Figure 4. The plots of the means and standard deviation. Asterisks show where statistically significant differences are, $p<0.01$ ( $p, \mathrm{p}$ value).

Red: the value obtained by dividing "the length from the lower end plate of the vertebra to the upper rims of the lumbar vessels" by "the length of the vertebra"

Black: the value obtained by dividing "the length from the lower end plates of the vertebra to the lower rims of the lumbar vessels" by "the length of the vertebra"

$\mathrm{A}$, anterior quarter slice; $\mathrm{C}$, center slice; $\mathrm{P}$, posterior quarter slice

within $8 \mathrm{~mm}$ from the end plate. For the findings in the current study, it is considered that the risk of damaging the lumbar artery from other vertebral bodies during insertion, fixation, and removal of the retractor is high. Lumbar vein can be identified easily without using a contrast agent in MRIs performed before surgery, as it keeps the invasiveness low and is considered very useful. A detailed preoperative evaluation should be performed for each case, and it is necessary to pay close attention so as not to damage the vessels when inserting a screw to fix the retractor at the time of LLIF. We measured the distance from the vertebral end plate of the lumbar artery before surgery and set it as one of the indicators of the safety zone when inserting the retractor during surgery. Seven lumbar vessels are located on vertebral disc level using sagittal MRI images. Beckman ${ }^{15)}$ reported that contralateral psoas hematoma was present in $0.18 \%$ of 3590 patients after LLIF. In LLIF, the contralateral side of the disc is removed with a curette, when vessels on the disc are vulnerable. Their reports are consistent with our results.

As for the limitations of this study, it was difficult to distinguish between the lumbar artery and vein, and blood vessels between vertebral bodies connecting between lumbar veins were difficult to identify. Diagnostic values are low when compared with CT using contrast media.

\section{Conclusion}

Because the lumbar artery is not necessarily located at the center of the vertebral body and many individual differences occur, it is thought that it is necessary to investigate the location of the lumbar artery more carefully in order to avoid lumbar arterial injury in LLIF surgery.

Conflicts of Interest: The authors declare that there are no relevant conflicts of interest.

Author Contributions: Masato Kiyohara wrote and prepared the manuscript, and all of the authors participated in the study design. All authors have read, reviewed, and approved the article.

Informed Consent: Informed consent was obtained from all participants in this study.

\section{References}

1. Kueper J, Fantini GA, Walker BR, et al. Incidence of vascular complications during lateral lumbar interbody fusion: an examination of the mini-open access technique. Eur Spine J. 2015;24(4): 800-9.

2. Mehren C, Mayer HM, Zandanell C, et al. The oblique anterolateral approach to the lumbar spine provides access to the lumbar spine with few early complications. Clin Orthop Relat Res. 2016; 474(9):2020-7.

3. Silvestre C, Mac-Thiong JM, Hilmi R, et al. Complications and morbidities of mini-open anterior retroperitoneal lumbar interbody fusion: Oblique lumbar interbody fusion in 179 patients. Asian Spine J. 2012;6(2):89-97.

4. Rodgers WB, Gerber EJ, Patterson J. Intraoperative and early 
postoperative complications in extreme lateral interbody fusion: an analysis of 600 cases. Spine (Phila Pa 1976). 2011;36(1):26-32.

5. Baker JK, Reardon PR, Reardon MJ, et al. Vascular injury in anterior lumbar surgery. Spine (Phila Pa 1976). 1993;18(15):2227-30.

6. Brau SA. Mini-open approach to the spine for anterior lumbar interbody fusion: description of the procedure, results and complications. Spine J. 2002;2(3):216-23.

7. Brau SA, Delamarter RB, Schiffman ML, et al. Vascular injury during anterior lumbar surgery. Spine J. 2004;4(4):409-12.

8. Fantini GA, Pappou IP, Girardi FP, et al. Major vascular injury during anterior lumbar spinal surgery: incidence, risk factors, and management. Spine (Phila Pa 1976). 2007;32(24):2751-8.

9. Quraishi NA, Konig M, Booker SJ, et al. Access related complications in anterior lumbar surgery performed by spinal surgeons. Eur Spine J. 2013;22(suppl 1):S16-20.

10. Rothenfluh DA, Koenig M, Stokes OM, et al. Access-related complications in anterior lumbar surgery in patients over 60 years of age. Eur Spine J. 2014;23(suppl 1):S86-92.

11. Kanda $Y$. Investigation of the freely available easy-to-use software 'EZR' for medical statistics. Bone Marrow Transplantation. Bone Marrow Transplant. 2013;48(3):452-8.
12. Karunanayake AL, Pathmeswaran A. Anatomical variations of lumbar arteries and their clinical implications: A cadaveric study. Hindawi Publishing Corporation ISRN Anatomy. 2013;2013: 154625.

13. Ratcliffe JF. The arterial anatomy of the adult human lumbar vertebral body: A microarteriographic study. J Anat. 1980;131(Pt 1): 57-79.

14. Orita S, Inage K, Sainoh $T$, et al. Lower lumbar segmental arteries can intersect over the intervertebral disc in the oblique lateral interbody fusion approach with a risk for arterial injury: Radiological analysis of lumbar segmental arteries by using magnetic resonance imaging. Spine (Phila Pa 1976). 2017;42(3):135-42.

15. Beckman JM, Vincent B, Park MS, et al. Contralateral psoas hematoma after minimally invasive, lateral retroperitoneal transpsoas lumbar interbody fusion: A multicenter review of 3950 lumbar levels. J Neurosurg Spine. 2017;26(1):50-4.

Spine Surgery and Related Research is an Open Access journal distributed under the Creative Commons Attribution-NonCommercial-NoDerivatives 4.0 International License. To view the details of this license, please visit (https://creativeco mmons.org/licenses/by-nc-nd/4.0/) 